\title{
On some distance measures of complex Pythagorean fuzzy sets and their applications in pattern recognition
}

\author{
Kifayat Ullah ${ }^{1} \cdot$ Tahir Mahmood $^{1} \cdot$ Zeeshan Ali $^{1} \cdot$ Naeem Jan $^{1}$
}

Received: 18 November 2018 / Accepted: 4 April 2019 / Published online: 17 May 2019

(c) The Author(s) 2019

\begin{abstract}
The concept of complex fuzzy set (CFS) and complex intuitionistic fuzzy set (CIFS) is two recent developments in the field of fuzzy set (FS) theory. The significance of these concepts lies in the fact that these concepts assigned membership grades from unit circle in plane, i.e., in the form of a complex number instead from $[0,1]$ interval. CFS cannot deal with information of yes and no type, while CIFS works only for a limited range of values. To deal with these kinds of problems, in this article, the concept of complex Pythagorean fuzzy set (CPFS) is developed. The novelty of CPFS lies in its larger range comparative to CFS and CIFS which is demonstrated numerically. It is discussed how a CFS and CIFS could be CPFS but not conversely. We investigated the very basic concepts of CPFSs and studied their properties. Furthermore, some distance measures for CPFSs are developed and their characteristics are studied. The viability of the proposed new distance measures in a building material recognition problem is also discussed. Finally, a comparative study of the proposed new work is established with pre-existing study and some advantages of CPFS are discussed over CFS and CIFS.
\end{abstract}

Keywords Complex fuzzy set · Complex intuitionistic fuzzy set · Complex Pythagorean fuzzy set · Distance measures

\section{Introduction}

Handling of uncertain and imprecise information has always been a challenge. Many theories are presented to cope with imprecision and uncertainty that exists in almost all the reallife problems such as theory of soft sets [1], theory of rough sets [2], and theory of FSs [3]. All these theories have their own characteristics and advantages, but among these Zadeh's FS is a remarkable concept and is greatly utilized in many situations of uncertainties including decision-making problems, pattern recognition, clustering, networking, and many other fields of computer and engineering. Zadeh's FS cope with uncertain events or objects by describing them in terms

Zeeshan Ali

zeeshan.msma434@iiu.edu.pk

Kifayat Ullah

kifayat.phdma72@iiu.edu.pk

Tahir Mahmood

tahirbakhat@iiu.edu.pk

Naeem Jan

naeem.phdma73@iiu.edu.pk

1 Department of Mathematics and Statistics, International Islamic University Islamabad, Islamabad 44000, Pakistan of a membership grades ranges on a scale of zero to one. This type of mathematical modeling enables scientists to describe the imprecision of an object or event numerically. An FS only allows us to describe the membership grade of an object, i.e., the degree of satisfaction; however, it does not provide any information about degree of dissatisfaction. In FS theory, if an object has a grade of membership as 0.7 , then its non-membership grade is chosen by default as $1-0.7=0.3$. Hence, an FS does not allow us to choose the non-membership grade independently. Realizing this, Atanassov [4] developed the notion of intuitionistic fuzzy set (IFS) which not only describes the membership grade of an object, but also describes its non-membership grade on a scale of zero to one, independently. Keeping the sum of both membership as well as non-membership between 0 and 1. Atanassov's IFS improved the concept of FS by facilitating the scientists in assigning membership and non-membership grades independently. However, an IFS somehow restricts us in a certain range, i.e., one cannot choose the membership and non-membership grades as 0.6 and 0.7 simultaneously, because their sum exceeds the unit interval. Realizing this, Yager [5] developed the theory of Pythagorean fuzzy set (PFS) which is also based on a membership and non-membership grade but with an 
Table 1 Comparison of constraints on ranges of IFS and PFS

\begin{tabular}{ll}
\hline Structure & Constraint \\
\hline IFS & $0 \leq M+N \leq 1$ \\
PFS & $0 \leq M^{2}+N^{2} \leq 1$ \\
\hline
\end{tabular}

improved constraint, i.e., the sum of squares of membership and non-membership grades must not exceed 1 . If we denote the membership and non-membership grades by $M$ and $N$, respectively, then the constraint on IFS and PFS is given in Table 1.

In [6], Ramot et al. proposed a new concept of CFS, where the membership grade of an element is a complex number from unit circle instead of a real number from $[0,1]$ interval. A CFS improved the concept of ordinary FS due to its larger range of membership grades. Following the concept of CFS, Alkouri et al. [7] developed the framework of CIFS which is basically based on two complex functions denoting the membership and non-membership grade of an object/element. Keeping in mind the significance of these two concepts, scientists contributed in these directions extensively. Ma et al. [8] developed CFS-based method to solve problems having multiple periodic factors. Dick et al. [9] studied some operations of CFS and Liu and Zhang [10] improved the results of defined by Dick et al. [9]. Greenfield et al. [11] proposed a novel concept of complex interval valued FS (CIVFS) which certainly improved the idea of CFS and generalizes the framework of interval valued fuzzy set (IVFS). Garg and Rani [12] proposed some aggregation operators for CIFSs and utilized those operators in multiattribute decision making (MADM). Some distance measures for complex intuitionistic fuzzy soft sets (CIFSSs) are developed by Kumar and Bajaj in [13], whereas the theory of power aggregation operators for CIFSs is proposed by Rani and Garg [14] which was further utilized in MADM. Singh et al. [15] studied the lattices of interval valued complex fuzzy sets and their granular decompositions. Selvachandran et al. [16] developed the notion of complex vague soft sets (CVSSs) and examined their entropy measures. Hu et al. [17] proposed some distance measures for CFSs and studied the continuity of operations of CFSs. Some similarity measures of CVSSs are proposed by Selvachandran et al. [18] and their applications in pattern recognitions are studied. In [19], Quek and Selvachandran studied the algebraic structures of CIFS associated with groups, while [20] discussed the application of CFS in E-commerce. Complex fuzzy concept lattice is studied in [21] and the idea of interval valued complex fuzzy soft set along with their applications is discussed in [22]. In [23], the authors studied Malaysian economy using interval valued complex fuzzy soft set. Some other study on CIFS can be found in $[24,25]$.

Distance and similarity measures are among the affective tools that have been utilized in FS theory and its general- ized forms to cope with problems of pattern recognition, clustering, MADM, and medical diagnosis. Since the evolution of FS theory, several types of distance and similarity measures have been introduced for FS, IFS as well as PFSs. Ngan et al. [26] proposed some $H$-max distance measures for IFSs and unitized those distance measures in decision making. Mishra et al. [27] assessed some cellular mobile service providing companies using the similarity measures of IFSs. Shen et al. [28] assessed the problem involving credit risk evaluation of partners using new similarity measures of IFSs in an extended TOPSIS method. Some Jaccard index-based similarity measures of IFSs are proposed by Hwang et al. [29] which are further utilized in a clustering problem. Luo and Zhao [30] defined a new distance measure for IFSs and applied these distance measures in a medical diagnosis problem. Li and Zeng [31] developed a similarity measure for PFS that is based on four kinds of membership functions and examined its viability and practicability. The cosine functionbased similarity measure for PFSs is defined by Wei and Wei [32] and its applications are studied. A number of distance and similarity measures of PFSs are developed by Zeng et al. [33] which were further utilized in MADM problem. Some point operator-based similarity measures of PFSs are developed by Biswas and Sarkar [34], and an MADM problem is solved using the proposed similarity measures. Some similarity measures for IFSs are studied by Garg [35-37] and their viability is demonstrated. Some distance measures of type 2 FS are developed in [38], while in [39], similarity measures for connection numbers based on set pair analysis are developed. These similarity measures are utilized in MADM problems. Ullah et al. [40] developed few similarity measures for T-spherical fuzzy set and utilized those similarity measures in building material recognition problems. [41-43] discussed some MADM problems in bipolar-valued hesitant fuzzy environments, while $[44,45]$ are based on some medical diagnosis and MADM problems based on Tspherical fuzzy and linguistic cubic fuzzy information. A state of art study of PFS is established in [46] and the study of information measures of PFSs is developed in [47]. Choquet integrals for PFSs are developed in [48], while some aggregation operators for PFSs are developed in [49]. Hesitant Pythagorean fuzzy McLaurin symmetric mean operators are developed and utilized in MADM in [50]. Some logarithmic aggregation operators of PFS and their applications are studied in [51]. Some generalized power aggregation operators for IFSs and their applications in MADM are studied in [52]. In [53], the correlation coefficients for IFSs are developed and applied in MADM. Some other related work can be found in [54-57].

The concept of FS proposed by Zadeh [3] and IFS proposed by Atanassov [4] discuss the uncertainties of imprecise events using real numbers as membership grades. However, neither FS nor IFS discussed the fractional ignorance and 
variations that exist in the data, for example, phase change or periodicity. Due to this reason, the idea of CFS has been introduced that extended the idea of FS from real membership grades to complex membership grade, therefore, lessened the chances of information loss. CIFS proposed by Alkouri et al. [7] improved the idea of CFS by adding another function to it known as non-membership function. It is discussed that the existing definition of CIFS did not describe the hesitancy degree and it needs to be redefined. Furthermore, CIFS cannot not describe some situations due to a restriction that the sum of membership and non-membership grades cannot exceed 1. A detailed analysis of the restrictions of CFS and CIFS has been done in Sect. 3. Therefore, in this paper, our aim is to develop the idea of CPFS. The novelty and effectiveness of proposed CPFS are demonstrated with the help of some examples and through a comparative study. Furthermore, motivated by the work of $[6,7]$, some distance measures of CPFSs are defined and their properties are investigated.

This article organized in Sect. 1 is based on introduction. In Sect. 2, some pre-requisites related to IFS, PFS, CFS, and CIFS are described along with an introduction to distance measures. In Sect. 3, the novelty of CPFS is demonstrated with the help of numerical examples and some basic operations are developed. Section 4 is based on distance measures for CPFSs and their characteristics. In Sect. 5, a pattern recognition problem is solved using the newly defined distance measures. Section six is based on a comparative study, while in Sect. 7, the advantages of CPFSs are discussed. Section 8 is based on some conclusive remarks and future directions.

\section{Preliminaries}

This section aims to provide a short literature survey of preexisting concepts related to IFS, PFS, CFS, and CIFS along with some other notions.

Definition 1 [4] An IFS $I$ is defined as $I=$ $\left\{\left(T_{I}(x), F_{I}(x)\right): x \in X\right\}$, where $T_{I}$ and $F_{I}$ denote the grades of membership and non-membership, respectively, with a constraint $0 \leq T_{I}(x)+F_{I}(x) \leq 1$. Moreover, the term $R_{I}=1-\left(T_{I}+F_{I}\right)$ is referred as hesitancy degree and $\left(T_{I}, F_{I}\right)$ is considered as intuitionistic fuzzy number (IFN).

Definition 2 [5] A PFS $I$ is defined as $I=$ $\left\{\left(T_{I}(x), F_{I}(x)\right): x \in X\right\}$, where $T_{I}$ and $F_{I}$ denote the grades of membership and non-membership, respectively, with a constraint $0 \leq T_{I}^{2}(x)+F_{I}^{2}(F) \leq 1$. Moreover, the term $R_{I}=\sqrt{1-\left(T_{I}^{2}+F_{I}^{2}\right)}$ is referred as hesitancy degree and $\left(T_{I}, F_{I}\right)$ is considered as Pythagorean fuzzy number (PyFN).
Definition 3 [6] ACFS $C$ is defined as: $C=\{(x$, $\left.\left.M_{C}(x)\right) / x \in X t\right\}$, where $M_{C}: U \rightarrow\{z: z \in C,|z| \leq 1\}$ and $M_{C}(x)=a+i b=T_{C}(x) \cdot \mathrm{e}^{2 \Pi i \cdot W_{C}(x)}$. Here, $T_{C}$ $(x)=\sqrt{a^{2}+b^{2}} \in \mathbb{R}$ and $T_{C}(x), W_{C}(x) \in[0,1]$, where $i=\sqrt{-1}$.

The range of membership grades of a CFS is demonstrated in Fig. 1. Where the points inside the circle represents all the complex numbers whose magnitudes lies between 0 and 1 .

Definition 4 [7] ACIFS $C$ is defined as: $C=$ $\left\{\left(x, M_{C}(x), N_{C}(x)\right) / x \in X\right\}$, where $M_{C}: U \rightarrow$ $\left\{z_{1}: z_{1} \in C,\left|z_{1}\right| \leq 1\right\} N_{C}: U \rightarrow\left\{z_{2}: z_{2} \in C,\left|z_{2}\right| \leq 1\right\}$, such that $M_{C}(x)=z_{1}=a_{1}+i b_{1}$ and $N_{C}(x)=z_{2}=a_{2}+i b_{2}$ provided that $0 \leq\left|z_{1}\right|+\left|z_{2}\right| \leq 1$ or $M_{C}(x)=T_{C}$ $(x) \cdot \mathrm{e}^{2 \Pi i \cdot W_{T_{C}}(x)}$ and $N_{C}(x)=F_{C}(x) \cdot \mathrm{e}^{2 \Pi i \cdot W_{F_{C}}(x)}$ satisfying the conditions: $0 \leq T_{C}(x)+F_{C}(x) \leq 1$ and $0 \leq W_{T_{C}}(x)+W_{F_{C}}(x) \leq 1$. Furthermore, $\tau=$ $\left(T \cdot \mathrm{e}^{2 \Pi \bar{i} \cdot W_{T}}, F \cdot \mathrm{e}^{2 \Pi i \cdot W_{F}}\right)$ is called complex intuitionistic fuzzy number (CIFN).

This definition of CIFS does not provide any information about the hesitancy degree of an element as an ordinary IFS. Therefore, this definition needs a little modification. An improved definition of CIFS is proposed in Sect. 3.

Furthermore, the novelty and significance of CIFS lie in the fact that it described an event having imprecision with the help of two complex numbers denoting the degree of membership and non-membership, respectively. The geometrical representation of the range of CIFS is similar to that of CFS depicted in Fig. 1. The following proposition proposed by Alkouri and Salleh [7] shows the novelty and superiority of CIFS.

Proposition 1 [7] Every CFS can be considered as CIFS but not conversely.

Now, some basic notions including complement, equality and inclusion of CFSs as well as CIFSs are presented. These notions provide bases for the new proposed work.

Definition 5 [7] For a two CIFNs $A=\left\{T_{A}(x) \cdot \mathrm{e}^{2 \Pi i \cdot W_{T_{A}}(x)}\right.$, $\left.F_{A}(x) \cdot \mathrm{e}^{2 \Pi i \cdot W_{F_{A}}(x)}\right\}$ and $B=\left\{T_{B}(x) \cdot \mathrm{e}^{2 \Pi i \cdot W_{T_{B}}(x)}, F_{B}\right.$ $\left.(x) \cdot \mathrm{e}^{2 \Pi i \cdot W_{F_{B}}(x)}\right\}$

1. $A \subseteq B$ if $T_{A}(x) \leq T_{B}(x), F_{A}(x) \geq F_{B}(x)$ and $W_{T_{A}}$ $(x) \leq W_{T_{B}}(x), W_{F_{A}}(x) \geq W_{F_{B}}(x)$.

2. $A=B$ iff $T_{A}(x)=T_{B}(x), F_{A}(x)=F_{B}(x)$ and $W_{T_{A}}$ $(x)=W_{T_{B}}(x), W_{F_{A}}(x)=W_{F_{B}}(x)$.

3. $A^{c}=\left\{F_{A}(x) \cdot \mathrm{e}^{2 \Pi i \cdot W_{F_{A}}(x)}, T_{A}(x) \cdot \mathrm{e}^{2 \Pi i \cdot W_{T_{A}}(x)}\right\}$.

The man key feature of CFS and CIFS is that these frameworks describe the uncertainty in a way that the chances of 


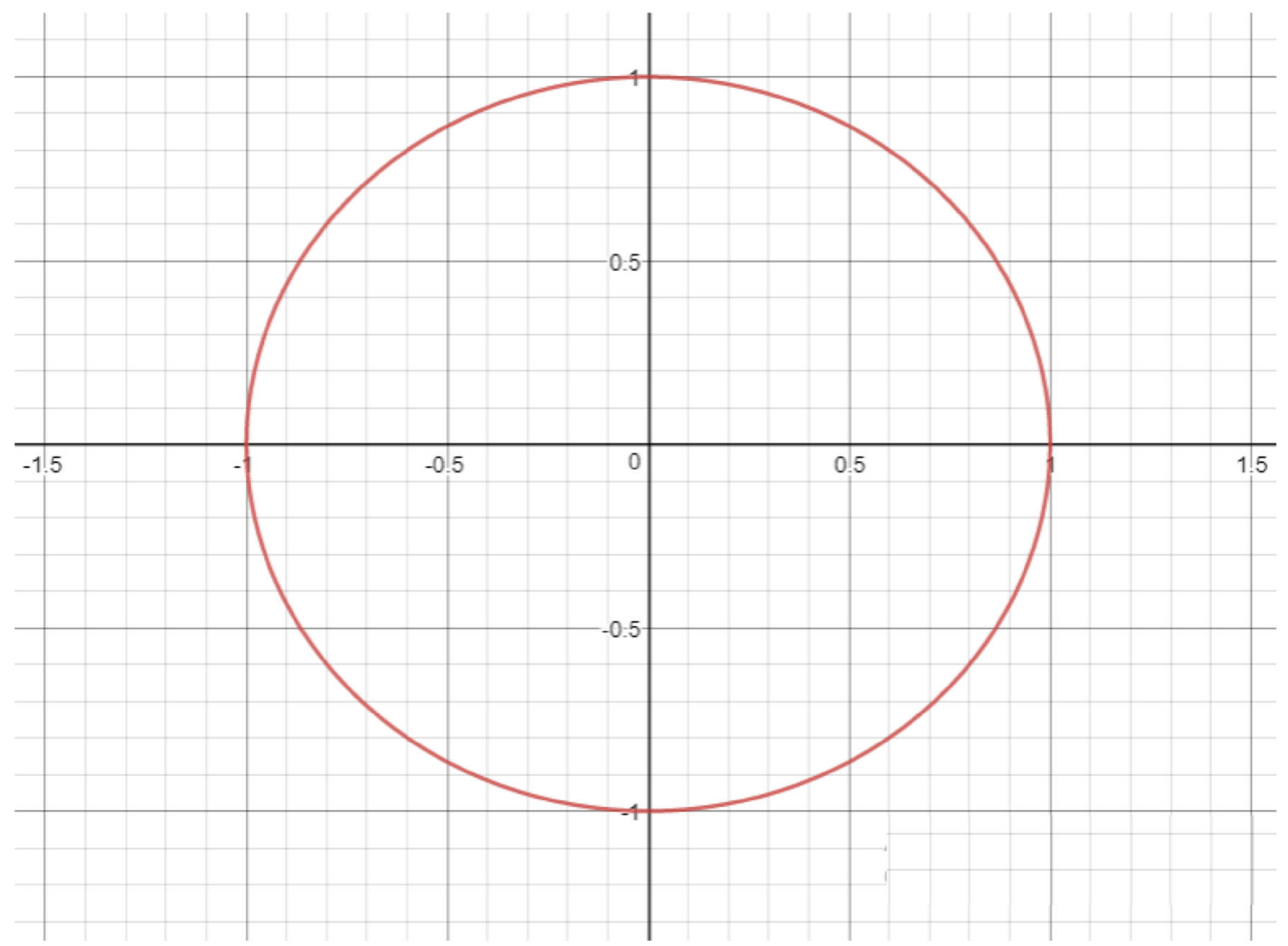

Fig. 1 Range of complex fuzzy set

losing information such as periodicity or phase shift, etc. It is noted that the existing definition of CIFS does not discuss the hesitancy degree of an uncertain event, as demonstrated in Sect. 2. Furthermore, the framework of CIFS has its limitations in its range, as in some cases, the sum of membership and non-membership grades exceeds 1 . To overcome this limitation, we propose the concept of CPFS.

\section{Complex Pythagorean fuzzy set}

In this section, we define CPFS and describe its novelty using some numerical examples along with some geometrical interpretations. It is also explained that a CFS and CIFS can be regarded as CPFS; however, converse is not true. Some basic operations of CPFSs are also proposed and supported with examples.

First, we redefine the CIFS, as the existing definition provides no information about the hesitancy degree of CIFS.

Definition 6 ACIFS $C$ is defined as: $C=$ $\left\{\left(x, M_{C}(x), N_{C}(x)\right) / x \in X\right\}$, where $M_{C}: U \rightarrow$ $\left\{z_{1}: z_{1} \in C,\left|z_{1}\right| \leq 1\right\} N_{C}: U \rightarrow\left\{z_{2}: z_{2} \in C,\left|z_{2}\right| \leq 1\right\}$, such that $M_{C}(x)=z_{1}=a_{1}+i b_{1}$ and $N_{C}(x)=z_{2}=a_{2}+i b_{2}$ provided that $0 \leq\left|z_{1}\right|+\left|z_{2}\right| \leq 1$ or $M_{C}(x)=T_{C}$ $(x) \cdot \mathrm{e}^{2 \Pi i \cdot W_{T_{C}}(x)}$ and $N_{C}(x)=F_{C}(x) \cdot \mathrm{e}^{2 \Pi i \cdot W_{F_{C}}(x)}$ satisfying the conditions: $0 \leq T_{C}(x)+F_{C}(x) \leq 1$ and $0 \leq W_{T_{C}}$ $(x)+W_{F_{C}}(x) \leq 1$. The term $H_{C}(x)=R \cdot \mathrm{e}^{2 \Pi i \cdot W_{R}}$, such that $R=1-\left(\left|z_{1}\right|+\left|z_{2}\right|\right)$ and $W_{R}(x)=1-\left(W_{T_{C}}(x)+W_{F_{C}}(x)\right)$ is considered as hesitancy degree of $x$. Furthermore, $C=$ $\left(T \cdot \mathrm{e}^{2 \Pi i \cdot W_{T}}, F \cdot \mathrm{e}^{2 \Pi i \cdot W_{F}}\right)$ is called CIFN.

Definition 7 ACPFS $C$ is defined as $C=$ $\left\{\left(x, M_{C}(x), N_{C}(x)\right) / x \in X\right\}$, where $M_{C}: U \rightarrow$ $\left\{z_{1}: z_{1} \in C,\left|z_{1}\right| \leq 1\right\} N_{C}: U \rightarrow\left\{z_{2}: z_{2} \in C,\left|z_{2}\right| \leq 1\right\}$, such that $M_{C}(x)=z_{1}=a_{1}+i b_{1}$ and $N_{C}(x)=z_{2}=a_{2}+i b_{2}$ provided that $0 \leq\left|z_{1}\right|^{2}+\left|z_{2}\right|^{2} \leq 1$ or $M_{C}(x)=T_{C}$ $(x) \cdot \mathrm{e}^{2 \Pi i \cdot W_{T_{C}}(x)}$ and $N_{C}(x)=F_{C}(x) \cdot \mathrm{e}^{2 \Pi i \cdot W_{F_{C}}(x)}$ satisfying the conditions: $0 \leq T_{C}^{2}(x)+F_{C}^{2}(x) \leq 1$ and $0 \leq W_{T_{C}}^{2}(x)+W_{F_{C}}^{2}(x) \leq 1$. Moreover, the term $H_{C}$ $(x)=R \cdot \mathrm{e}^{2 \Pi i \cdot W_{R_{c}}(x)}$, such that $R=\sqrt{1-\left(\left|z_{1}\right|+\left|z_{2}\right|\right)}$ and $W_{R}(x)=\sqrt{1-\left(W_{T_{C}}(x)+W_{F_{C}}(x)\right)}$ is considered as hesitancy degree of $x$. Furthermore, $C=$ $\left(T \cdot \mathrm{e}^{2 \Pi i \cdot W_{T}}, F \cdot \mathrm{e}^{2 \Pi i \cdot W_{F}}\right)$ is called CPyFN. 
The membership and non-membership grades of CPFS are clearly complex numbers in polar/Cartesian form. These two types of notations are interconvertible as follows:

$$
\begin{aligned}
M_{C}(x) & =T_{C}(x) \cdot \mathrm{e}^{2 \Pi i \cdot W_{T_{C}}(x)} \\
& =T_{C}(x) \cdot\left(\operatorname{Cos} 2 \Pi W_{T_{C}}(x)+i \operatorname{Sin} 2 \Pi W_{T_{C}}(x)\right) \\
& =a_{1}+i b_{1}=z_{1}, \\
N_{C}(x) & =F_{C}(x) \cdot \mathrm{e}^{2 \Pi i \cdot W_{F_{C}}(x)} \\
& =F_{C}(x) \cdot\left(\operatorname{Cos} 2 \Pi W_{F_{C}}(x)+i \operatorname{Sin} 2 \Pi W_{F_{C}}(x)\right) \\
& =a_{2}+i b_{2}=z_{2} .
\end{aligned}
$$

The reason to develop the concept of CPFS is that in some cases information could not be processed using CIFS and CFS. Already in [7], the authors proved the generalization and superiority of CIFS over CFS with the help of some results and examples. Here we demonstrate the limitation of CIFS and established the superiority of CPFS.

Consider an example of CIFS of the form $\{(x$, $(0.399134+0.0263 i), \quad(0.399134+0.0263 i) 0.4 \mathrm{e}^{i 2 \Pi(0.6)}$, $\left.\left.0.5 \mathrm{e}^{i 2 \Pi(0.4)}\right)\right\}$. This set satisfies the basic definition of CIFS as $|0.399134+0.0263 \mathrm{i}|=0.4$ and $|0.499519+0.021925 \mathrm{i}|=0.5$ and $0 \leq 0.4+0.5 \leq 1$. The polar form of this CIFN is $\left\{\left(x, 0.4 \mathrm{e}^{i 2 \Pi(0.6)}, 0.5 \mathrm{e}^{i 2 \Pi(0.4)}\right)\right\}$.

On the other hand, consider the representation of an uncertain event as $\{(x, 0.698948+0.038363 \mathrm{i}, 0.597693+$ $0.05257 \mathrm{i})\}$. Then, $|0.698948+0.038363 \mathrm{i}|=0.7$ and $|0.597693+0.05257 \mathrm{i}|=0.6$ and $0 \leq 0.7+0.6 \$ 1$. This means that CIFS is not enough to deal with this type of information. However, the concept of CPFS can handle such information $0 \leq 0.7^{2}+0.6^{2}=0.85 \leq 1$. Hence, the number, $\{(x, 0.698948+0.038363 \mathrm{i}, 0.597693+0.05257 \mathrm{i})\}$ which can be written as $\left\{\left(x, 0.7 \mathrm{e}^{i 2 \Pi(0.5)}, 0.6 \mathrm{e}^{i 2 \Pi(0.8)}\right)\right\}$, is considered as a CPyFN.

Figure 2 shows the comparison of the restrictions of CIFS and CFS.

Now, the basic operations such as inclusion, complement, and equality of CPFSs are presented which are analogous to operations of CIFSs and CFSs.

Proposition 2 Every CIFS can be considered as CPFS but not conversely.

Proof Straightforward from Definitions 6 and 7.

Definition 8 For a two CPyFNs $A=$ $\left\{T_{A}(x) \cdot \mathrm{e}^{2 \Pi i \cdot W_{T_{A}}(x)}, F_{A}(x) \cdot \mathrm{e}^{2 \Pi i \cdot W_{F_{A}}(x)}\right\}$ and $B=$
$\left\{T_{B}(x) \cdot \mathrm{e}^{2 \Pi i \cdot W_{T_{B}}(x)}, F_{B}(x) \cdot \mathrm{e}^{2 \Pi i \cdot W_{F_{B}}}(x)\right\}$, then

1. $A \subseteq B$ if $T_{A}(x) \leq T_{B}(x), F_{A}(x) \geq F_{B}(x)$ and $W_{T_{A}}$ $(x) \leq W_{T_{B}}(x), W_{F_{A}}(x) \geq W_{F_{B}}(x)$.

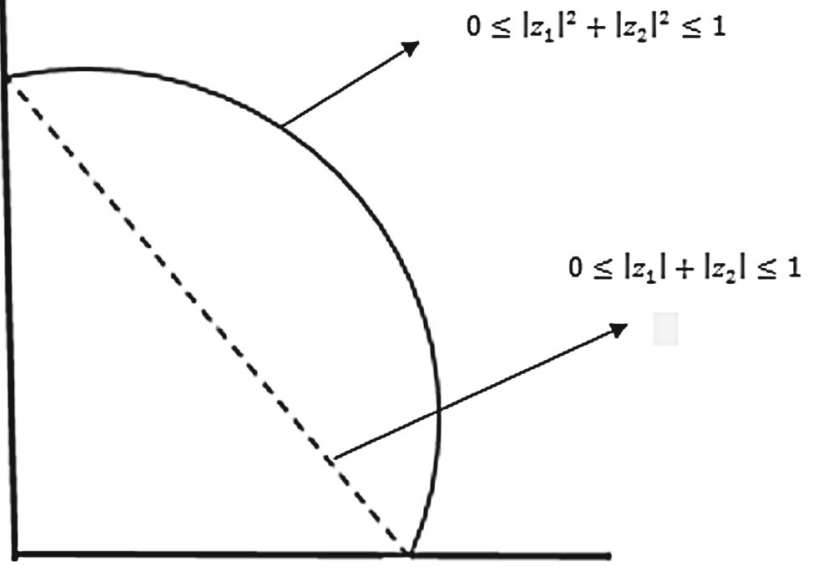

Fig. 2 Comparisons of restrictions of CIFS and CPFS

2. $A=B$ iff $T_{A}(x)=T_{B}(x), F_{A}(x)=F_{B}(x)$ and $W_{T_{A}}$ $(x)=W_{T_{B}}(x), W_{F_{A}}(x)=W_{F_{B}}(x)$.

3. $A^{c}=\left\{F_{A}(x) \cdot \mathrm{e}^{2 \Pi i \cdot W_{F_{A}}(x)}, T_{A}(x) \cdot \mathrm{e}^{2 \Pi i \cdot W_{T_{A}}(x)}\right\}$.

Definition 9 Ascore function $S$ and accuracy function $H$ on $\tau=\left(T \cdot \mathrm{e}^{2 \Pi i \cdot W_{T}}, F \cdot \mathrm{e}^{2 \Pi i \cdot W_{F}}\right)$ is defined as:

$S(\tau)=(T-F)+\frac{1}{2 \Pi}\left(2 \Pi \cdot W_{T}-2 \Pi \cdot W_{F}\right)$

$H(\tau)=(T+F)+\frac{1}{2 \Pi}\left(2 \Pi \cdot W_{T}+2 \Pi \cdot W_{F}\right)$,

where $S(\tau) \in[-2,2]$ and $H(\tau) \in[0,2]$.

Definition 10 An order relation between two CPyFNs $\tau$ and $\dot{\tau}$ is of the form:

1. If $S(\tau)>S(\dot{\tau})$, then $\tau>\dot{\tau}$; similarly, if $H(\tau)>H(\dot{\tau})$, then $\tau>\dot{\tau}$.

2. If $S(\tau)=S(\dot{\tau})$, then $\tau=\dot{\tau}$; similarly, if $H(\tau)=H(\dot{\tau})$ then $\tau=\dot{\tau}$.

\section{Distance measures of CPFSs}

The purpose of this section is to develop some DMs, WDMs, and GWDMs for CPFSs. Throughout this article, $\omega=$ $\left(\omega_{1}, \omega_{2}, \ldots, \omega_{n}\right)^{\mathrm{T}}$ represents the weight vector, where $\omega_{i} \in$ $[0,1], \sum_{i=1}^{n} \omega_{i}=1$, and $A, B, C$ be three CPFSs. Furthermore, $a_{i}, b_{i}, c_{i},, r_{i} \in[0,1]$, such that $a_{i}+b_{i}+c_{i}+r_{i}=1$. 
Definition 11 A DM for CPFS is defined as

$$
\begin{aligned}
& D_{\mathrm{CPyFS}}^{1}(A, B) \\
& =\frac{1}{2} \sum_{i=1}^{n}\left[\left(a_{1} \cdot\left|T_{A}^{2}\left(x_{i}\right)-T_{B}^{2}\left(x_{i}\right)\right|+b_{1} \cdot\left|F_{A}^{2}\left(x_{i}\right)-F_{B}^{2}\left(x_{i}\right)\right|\right.\right. \\
& \left.\quad+c_{1} \cdot \max \left(\left|T_{A}^{2}\left(x_{i}\right)-T_{B}^{2}\left(x_{i}\right)\right|,\left|F_{A}^{2}\left(x_{i}\right)-F_{B}^{2}\left(x_{i}\right)\right|\right)\right) \\
& \quad+\frac{1}{2 \Pi}\left(a_{2} \cdot\left|2 \Pi W_{T_{A}^{2}}\left(x_{i}\right)-2 \Pi W_{T_{B}^{2}}\left(x_{i}\right)\right|\right. \\
& +b_{2} \cdot\left|2 \Pi W_{F_{A}^{2}}\left(x_{i}\right)-2 \Pi W_{F_{B}^{2}}\left(x_{i}\right)\right| \\
& \quad+c_{2} \cdot \max \left(\left|2 \Pi W_{T_{A}^{2}}\left(x_{i}\right)-2 \Pi W_{T_{B}^{2}}\left(x_{i}\right)\right|\right. \\
& \left.\left.\left.\quad\left|2 \Pi W_{F_{A}^{2}}\left(x_{i}\right)-2 \Pi W_{F_{B}^{2}}\left(x_{i}\right)\right|\right)\right)\right]
\end{aligned}
$$
ties:

The above $D_{\mathrm{CPyFS}}^{1}(A, B)$ satisfies the following proper-

1. $0 \leq D_{\mathrm{CPyFS}}^{1}(A, B) \leq 1$

2. $D_{\mathrm{CPyFS}}^{1}(A, B)=D_{\mathrm{CPyFS}}^{1}(B, A)$.

3. $D_{\mathrm{CPyFS}}^{1}(A, B)=1$ if $A=B$, i.e., $T_{A}(x)=T_{B}(x), F_{A}$ $(x)=F_{B}(x)$ and $W_{T_{A}}(x)=W_{T_{B}}(x), W_{F_{A}}(x)=W_{F_{B}}$ $(x)$.

4. If $A \subseteq B \subseteq C$, then $D_{\mathrm{CPyFS}}^{1}(A, C)>D_{\mathrm{CPyFS}}^{1}(A, B)$ and $D_{\mathrm{CPyFS}}^{1}(A, C)>D_{\mathrm{CPyFS}}^{1}(B, C)$.

Proof The condition $0 \leq D_{\mathrm{CPyFS}}^{1}(A, B)$ obviously holds true. Next, consider

$$
\begin{aligned}
D_{\mathrm{CPyFS}}^{1}(A, B) \\
=\frac{1}{2} \sum_{i=1}^{n}\left[\left(a_{1} \cdot\left|T_{A}^{2}\left(x_{i}\right)-T_{B}^{2}\left(x_{i}\right)\right|+b_{1} \cdot\left|F_{A}^{2}\left(x_{i}\right)-F_{B}^{2}\left(x_{i}\right)\right|\right.\right. \\
\left.\quad+c_{1} \cdot \max \left(\left|T_{A}^{2}\left(x_{i}\right)-T_{B}^{2}\left(x_{i}\right)\right|,\left|F_{A}^{2}\left(x_{i}\right)-F_{B}^{2}\left(x_{i}\right)\right|\right)\right) \\
\quad+\frac{1}{2 \Pi}\left(a_{2} \cdot\left|2 \Pi W_{T_{A}^{2}}\left(x_{i}\right)-2 \Pi W_{T_{B}^{2}}\left(x_{i}\right)\right|\right. \\
\quad+b_{2} \cdot\left|2 \Pi W_{F_{A}^{2}}\left(x_{i}\right)-2 \Pi W_{F_{B}^{2}}\left(x_{i}\right)\right| \\
\quad+c_{2} \cdot \max \left(\left|2 \Pi W_{T_{A}^{2}}\left(x_{i}\right)-2 \Pi W_{T_{B}^{2}}\left(x_{i}\right)\right|,\right. \\
\left.\left.\left.\quad\left|2 \Pi W_{F_{A}^{2}}\left(x_{i}\right)-2 \Pi W_{F_{B}^{2}}\left(x_{i}\right)\right|\right)\right)\right] \\
=\frac{1}{2} \sum_{i=1}^{n}\left[\left(a_{1} \cdot 1+b_{1} \cdot 1+c_{1} \cdot \max (1,1)\right)\right. \\
\left.\quad+\frac{1}{2 \Pi}\left(a_{2} \cdot 2 \Pi+b_{2} \cdot 2 \Pi+c_{2} \cdot \max (2 \Pi, 2 \Pi)\right)\right] \\
=\frac{1}{2} \sum_{i=1}^{n}\left[\left(a_{1}+b_{1}+c_{1}\right)+\frac{2 \Pi}{2 \Pi}\left(a_{2}+b_{2}+c_{2}\right)\right] .
\end{aligned}
$$

As $a_{1}+b_{1}+c_{1}=1$ and $a_{2}+b_{2}+c_{2}=1$. Therefore,

$=\frac{1}{2} \sum_{i=1}^{n}\left[\left(a_{1}+b_{1}+c_{1}\right)+\frac{2 \Pi}{2 \Pi}\left(a_{2}+b_{2}+c_{2}\right)\right]=\frac{2}{2}=1$.

Therefore, $0 \leq D_{\mathrm{CPyFS}}^{1}(A, B) \leq 1$.

The conditions (2) and (3) are straightforward. To prove (4), using Definition 8, we have $1 \geq T_{A}^{2}\left(x_{i}\right) \geq T_{B}^{2}\left(x_{i}\right) \geq T_{C}^{2}$ $\left(x_{i}\right) \geq 0$ and $2 \Pi \leq 2 \Pi W_{T_{A}^{2}}\left(x_{i}\right) \leq 2 \Pi W_{T_{B}^{2}}\left(x_{i}\right) \leq 2 \Pi W_{T_{C}^{2}}$ $\left(x_{i}\right) \leq 0$. Therefore,

$\left|T_{A}^{2}\left(x_{i}\right)-T_{B}^{2}\left(x_{i}\right)\right| \leq\left|T_{A}^{2}\left(x_{i}\right)-T_{C}^{2}\left(x_{i}\right)\right|$

$\left|2 \Pi W_{T_{A}^{2}}\left(x_{i}\right)-2 \Pi W_{T_{B}^{2}}\left(x_{i}\right)\right| \leq\left|2 \Pi W_{T_{A}^{2}}\left(x_{i}\right)-2 \Pi W_{T_{C}^{2}}\left(x_{i}\right)\right|$

$\left|F_{A}^{2}\left(x_{i}\right)-F_{B}^{2}\left(x_{i}\right)\right| \leq\left|F_{A}^{2}\left(x_{i}\right)-F_{C}^{2}\left(x_{i}\right)\right|$

$\left|2 \Pi W_{F_{A}^{2}}\left(x_{i}\right)-2 \Pi W_{F_{B}^{2}}\left(x_{i}\right)\right| \leq\left|2 \Pi W_{F_{A}^{2}}\left(x_{i}\right)-2 \Pi W_{F_{C}^{2}}\left(x_{i}\right)\right|$.

Therefore, $D_{\mathrm{CPyFS}}^{1}(A, B) \leq D_{\mathrm{CPyFS}}^{1}(A, C)$. Similarly, $D_{\mathrm{CPyFS}}^{1}(B, C) \leq D_{\mathrm{CPyFS}}^{1}(A, C)$.

Definition 12 The DM of two CPFS is defined as:

$$
\begin{aligned}
& D_{\mathrm{CPyFS}}^{2}(A, B) \\
& =\frac{1}{2} \sum_{i=1}^{n}\left[\left(a_{1} \cdot\left|T_{A}^{2}\left(x_{i}\right)-T_{B}^{2}\left(x_{i}\right)\right|+b_{1} \cdot\left|F_{A}^{2}\left(x_{i}\right)-F_{B}^{2}\left(x_{i}\right)\right|\right.\right. \\
& \quad+r_{1} \cdot\left|R_{A}^{2}\left(x_{i}\right)-R_{B}^{2}\left(x_{i}\right)\right| \\
& \left.\quad+c_{1} \cdot \max \left(\left|T_{A}^{2}\left(x_{i}\right)-T_{B}^{2}\left(x_{i}\right)\right|,\left|F_{A}^{2}\left(x_{i}\right)-F_{B}^{2}\left(x_{i}\right)\right|,\left|R_{A}^{2}\left(x_{i}\right)-R_{B}^{2}\left(x_{i}\right)\right|\right)\right) \\
& \quad+\frac{1}{2 \Pi}\left(a_{2} \cdot\left|2 \Pi W_{T_{A}^{2}}\left(x_{i}\right)-2 \Pi W_{T_{B}^{2}}\left(x_{i}\right)\right|+b_{2} \cdot\left|2 \Pi W_{F_{A}^{2}}\left(x_{i}\right)-2 \Pi W_{F_{B}^{2}}\left(x_{i}\right)\right|\right. \\
& \quad+r_{2} \cdot\left|2 \Pi W_{R_{A}^{2}}\left(x_{i}\right)-2 \Pi W_{R_{B}^{2}}\left(x_{i}\right)\right| \\
& \quad+c_{2} \cdot \max \left(\left|2 \Pi W_{T_{A}^{2}}\left(x_{i}\right)-2 \Pi W_{T_{B}^{2}}\left(x_{i}\right)\right|,\left|2 \Pi W_{F_{A}^{2}}\left(x_{i}\right)-2 \Pi W_{F_{B}^{2}}\left(x_{i}\right)\right|,\right. \\
& \\
& \left.\left.\left|2 \Pi W_{R_{A}^{2}}\left(x_{i}\right)-2 \Pi W_{R_{B}^{2}}\left(x_{i}\right)\right|\right)\right] .
\end{aligned}
$$
ties:

The above $D_{\mathrm{CPyFS}}^{2}(A, B)$ satisfies the following proper-

1. $0 \leq D_{\mathrm{CPyFS}}^{2}(A, B) \leq 1$.

2. $D_{\mathrm{CPyFS}}^{2}(A, B)=D_{\mathrm{CPyFS}}^{2}(B, A)$.

3. $D_{\mathrm{CPyFS}}^{2}(A, B)=1$ if $A=B$, i.e., $T_{A}(x)=T_{B}(x), F_{A}$ $(x)=F_{B}(x), R_{A}(x)=R_{B}(x)$ and $W_{T_{A}}(x)=W_{T_{B}}(x)$, $W_{F_{A}}(x)=W_{F_{B}}(x), W_{R_{A}}(x)=W_{R_{B}}(x)$.

4. If $A \subseteq B \subseteq C$, then $D_{\mathrm{CPyFS}}^{2}(A, C)>D_{\mathrm{CPyFS}}^{2}(A, B)$ and $D_{\mathrm{CPyFS}}^{2}(A, C)>D_{\mathrm{CPyFS}}^{2}(B, C)$.

Now, the proposed DMs are further extended to weighted distance measure, because in real-life problems, the weight of the opinion of experts does matter sometimes. 
Definition 13 The WDM for CPFS is defined as

$$
\begin{aligned}
& W D_{\mathrm{CPyFS}}^{1}(A, B) \\
& =\frac{1}{2 \sum_{i=1}^{n} \omega_{i}}\left[\sum _ { i = 1 } ^ { n } \omega _ { i } \left[\left(a_{1} \cdot\left|T_{A}^{2}\left(x_{i}\right)-T_{B}^{2}\left(x_{i}\right)\right|+b_{1} \cdot\left|F_{A}^{2}\left(x_{i}\right)-F_{B}^{2}\left(x_{i}\right)\right|\right.\right.\right. \\
& \left.+c_{1} \cdot \max \left(\left|T_{A}^{2}\left(x_{i}\right)-T_{B}^{2}\left(x_{i}\right)\right|,\left|F_{A}^{2}\left(x_{i}\right)-F_{B}^{2}\left(x_{i}\right)\right|\right)\right) \\
& +\frac{1}{2 \Pi}\left(a_{2} \cdot\left|2 \Pi W_{T_{A}^{2}}\left(x_{i}\right)-2 \Pi W_{T_{B}^{2}}\left(x_{i}\right)\right|+b_{2} \cdot\left|2 \Pi W_{F_{A}^{2}}\left(x_{i}\right)-2 \Pi W_{F_{B}^{2}}\left(x_{i}\right)\right|\right. \\
& \left.\left.\left.+c_{2} \cdot \max \left(\left|2 \Pi W_{T_{A}^{2}}\left(x_{i}\right)-2 \Pi W_{T_{B}^{2}}\left(x_{i}\right)\right|,\left|2 \Pi W_{F_{A}^{2}}\left(x_{i}\right)-2 \Pi W_{F_{B}^{2}}\left(x_{i}\right)\right|\right)\right)\right]\right] .
\end{aligned}
$$

The above $W D_{\mathrm{CPyFS}}^{1}(A, B)$ satisfies the following properties:

1. $0 \leq W D_{\mathrm{CPyFS}}^{1}(A, B) \leq 1$.

2. $W D_{\mathrm{CPyFS}}^{1}(A, B)=W D_{\mathrm{CPyFS}}^{1}(B, A)$.

3. $W D_{\mathrm{CPyFS}}^{1}(A, B)=1$ if $A=B$, i.e., $T_{A}(x)=T_{B}(x), F_{A}$ $(x)=F_{B}(x)$ and $W_{T_{A}}(x)=W_{T_{B}}(x), W_{F_{A}}(x)=W_{F_{B}}$ $(x)$.

4. If $A \subseteq B \subseteq C$, then $W D_{\mathrm{CPyFS}}^{1}(A, C)>W D_{\mathrm{CPyFS}}^{1}$ $(A, B)$ and $W D_{\mathrm{CPyFS}}^{1}(A, C)>W D_{\mathrm{CPyFS}}^{1}(B, C)$.

Definition 14 The WDM for CPFS is defined as

$$
\begin{aligned}
& W D_{C P y F S}^{2}(A, B)=\frac{1}{2 \sum_{i=1}^{n} \omega_{i}} \\
& {\left[\sum _ { i = 1 } ^ { n } \omega _ { i } \left[\left(a_{1} \cdot\left|T_{A}^{2}\left(x_{i}\right)-T_{B}^{2}\left(x_{i}\right)\right|+b_{1} \cdot\left|F_{A}^{2}\left(x_{i}\right)-F_{B}^{2}\left(x_{i}\right)\right|+r_{1}\right.\right.\right.} \\
& \text { - }\left|R_{A}^{2}\left(x_{i}\right)-R_{B}^{2}\left(x_{i}\right)\right| \\
& \left.+c_{1} \cdot \max \left(\left|T_{A}^{2}\left(x_{i}\right)-T_{B}^{2}\left(x_{i}\right)\right| \cdot\left|F_{A}^{2}\left(x_{i}\right)-F_{B}^{2}\left(x_{i}\right)\right| \cdot\left|R_{A}^{2}\left(x_{i}\right)-R_{B}^{2}\left(x_{i}\right)\right|\right)\right) \\
& +\frac{1}{2 \Pi}\left(a_{2} \cdot\left|2 \Pi W_{T_{A}^{2}}\left(x_{i}\right)-2 \Pi W_{T_{B}^{2}}\left(x_{i}\right)\right|+b_{2} \cdot\left|2 \Pi W_{F_{A}^{2}}\left(x_{i}\right)-2 \Pi W_{F_{B}^{2}}\left(x_{i}\right)\right|\right. \\
& +r_{2} \cdot\left|2 \Pi W_{R_{A}^{2}}\left(x_{i}\right)-2 \Pi W_{R_{B}^{2}}\left(x_{i}\right)\right| \\
& +c_{2} \cdot \max \left(\left|2 \Pi W_{T_{A}^{2}}\left(x_{i}\right)-2 \Pi W_{T_{B}^{2}}\left(x_{i}\right)\right|\right. \text {, } \\
& \left.\left.\left.\left.\left|2 \Pi W_{F_{A}^{2}}\left(x_{i}\right)-2 \Pi W_{F_{B}^{2}}\left(x_{i}\right)\right|,\left|2 \Pi W_{R_{A}^{2}}\left(x_{i}\right)-2 \Pi W_{R_{B}^{2}}\left(x_{i}\right)\right|\right)\right)\right]\right] .
\end{aligned}
$$

The above $W D_{\mathrm{CPyFS}}^{2}(A, B)$ satisfies the following properties:

1. $0 \leq W D_{\mathrm{CPyFS}}^{2}(A, B) \leq 1$.

2. $W D_{\mathrm{CPyFS}}^{2}(A, B)=W D_{\mathrm{CPyFS}}^{2}(B, A)$.

3. $W D_{\mathrm{CPyFS}}^{2}(A, B)=1$ if $A=B$, i.e., $T_{A}(x)=T_{B}(x)$, $F_{A}(x)=F_{B}(x), R_{A}(x)=R_{B}(x)$ and $W_{T_{A}}(x)=W_{T_{B}}$ $(x), W_{F_{A}}(x)=W_{F_{B}}(x), W_{R_{A}}(x)=W_{R_{B}}(x)$.

4. If $A \subseteq B \subseteq C$, then $W D_{\mathrm{CPyFS}}^{2}(A, C)>W D_{\mathrm{CPyFS}}^{2}$ $(A, B)$ and $W D_{\mathrm{CPyFS}}^{2}(A, C)>W D_{\mathrm{CPyFS}}^{2}(B, C)$.

\section{Application}

The purpose of this section is to utilize the DMs developed in Sect. 4 in practical problems; here, we solve the famous building material recognition problem using developed DMs of CPFSs.

\section{Building material recognition problem}

In this type of problems, the aim is to identify the class of unknown building material using the degree of distance measure of unknown material with that of known building materials. The detailed steps of algorithm for finding the class of unknown building material are described as:

1. Obtained the information about the known building materials in the form of CPFSs.

2. Compute the distance measure of unknown material with known building material.

3. Rank the distance measures to find out the class of unknown material.

The following example is to demonstrate the building material recognition problem.

Example 1 Consider four building material, such as sealant, floor varnish, wall paint, and polyvinyl chloride flooring represented by four CPyFNs $A_{i}(i=1,2,3,4)$ with attribute set denoted by $X=\left\{x_{1}, x_{2}, x_{3}, x_{4}, x_{5}, x_{6}, x_{7}\right\}$ and the weight vector of attribute is $\omega=(0.11,0.14,0.1,0.18,0.21$, $0.10,0.16)^{\mathrm{T}}$. Let $A$ be the unknown building material, whose class is needed to be determine. The information about known and unknown building material in the form of CPyFNs is provided in Table 2. The detailed steps of this process are demonstrated as follows.

1. In this step, the decision makers provide their preferences about the known building materials with which the distance degree of unknown building material is to be determined. The information of the decision makers is given in Table 2.

2. This step involves the measurement of distance measures of each known material with that of unknown material. The weighted distance measures given in Eq. (3) are utilized to obtain the distance measure. The results obtained using Eq. (3) with $\left\{a_{1}=0.3, b_{1}=0.5, c_{1}=0.2\right\}$ and $\left\{a_{2}=0.1, b_{2}=0.2, c_{2}=0.7\right\}$ are provided in Table 3 .

Here, the distance measure defined in Eq. (4) is utilized to compute the distance measures between known and unknown building materials. The concept of $W D_{\mathrm{CPyFS}}^{2}\left(A_{i}, A\right), i=1$, 2, 3, 4 for CPFS is applied in Table 1. The results obtained using Eq. (4) with $\left\{a_{1}=0.3, b_{1}=0.4, c_{1}=0.2, r_{1}=0.1\right\}$ 
Table 2 Complex Pythagorean fuzzy information about known and unknown materials

\begin{tabular}{lllllll}
\hline & $A_{1}$ & $A_{2}$ & $A_{3}$ & $A_{4}$ & $A$ \\
\hline$x_{1}$ & $\left(0.8 \mathrm{e}^{i \cdot 2 \Pi(0.7)}, 0.3 \mathrm{e}^{i \cdot 2 \Pi(0.4)}\right)$ & $\left(0.7 \mathrm{e}^{i \cdot 2 \Pi(0.5)}, 0.4 \mathrm{e}^{i \cdot 2 \Pi(0.7)}\right)$ & $\left(0.5 \mathrm{e}^{i \cdot 2 \Pi(0.6)}, 0.7 \mathrm{e}^{i \cdot 2 \Pi(0.5)}\right)$ & $\left(0.7 \mathrm{e}^{i \cdot 2 \Pi(0.6)}, 0.3 \mathrm{e}^{i \cdot 2 \Pi(0.5)}\right)$ & $\left(1 \cdot \mathrm{e}^{i \cdot 2 \Pi}, 0\right)$ \\
$x_{2}$ & $\left(0.7 \mathrm{e}^{i \cdot 2 \Pi(0.6)}, 0.5 \mathrm{e}^{i \cdot 2 \Pi(0.6)}\right)$ & $\left(0.6 \mathrm{e}^{i \cdot 2 \Pi(0.7)}, 0.6 \mathrm{e}^{i \cdot 2 \Pi(0.5)}\right)$ & $\left(0.6 \mathrm{e}^{i \cdot 2 \Pi(0.5)}, 0.5 \mathrm{e}^{i \cdot 2 \Pi(0.6)}\right)$ & $\left(0.8 \mathrm{e}^{i \cdot 2 \Pi(0.5)}, 0.4 \mathrm{e}^{i \cdot 2 \Pi(0.6)}\right)$ & $\left(1 \cdot \mathrm{e}^{i \cdot 2 \Pi}, 0\right)$ \\
$x_{3}$ & $\left(0.9 \mathrm{e}^{i \cdot 2 \Pi(0.4)}, 0.2 \mathrm{e}^{i \cdot 2 \Pi(0.7)}\right)$ & $\left(0.8 \mathrm{e}^{i \cdot 2 \Pi(0.8)}, 0.3 \mathrm{e}^{i \cdot 2 \Pi(0.3)}\right)$ & $\left(0.3 \mathrm{e}^{i \cdot 2 \Pi(0.6)}, 0.3 \mathrm{e}^{i \cdot 2 \Pi(0.6)}\right)$ & $\left(0.7 \mathrm{e}^{i \cdot 2 \Pi(0.6)}, 0.5 \mathrm{e}^{i \cdot 2 \Pi(0.3)}\right)$ & $\left(1 \cdot \mathrm{e}^{i \cdot 2 \Pi}, 0\right)$ \\
$x_{4}$ & $\left(0.6 \mathrm{e}^{i \cdot 2 \Pi(0.6)}, 0.5 \mathrm{e}^{i \cdot 2 \Pi(0.5)}\right)$ & $\left(0.8 \mathrm{e}^{i \cdot 2 \Pi(0.9)}, 0.3 \mathrm{e}^{i \cdot 2 \Pi(0.1)}\right)$ & $\left(0.5 \mathrm{e}^{i \cdot 2 \Pi(0.7)}, 0.5 \mathrm{e}^{i \cdot 2 \Pi(0.5)}\right)$ & $\left(0.4 \mathrm{e}^{i \cdot 2 \Pi(0.8)}, 0.7 \mathrm{e}^{i \cdot 2 \Pi(0.2)}\right)$ & $\left(1 \cdot \mathrm{e}^{i \cdot 2 \Pi}, 0\right)$ \\
$x_{5}$ & $\left(0.5 \mathrm{e}^{i \cdot 2 \Pi(0.3)}, 0.6 \mathrm{e}^{i \cdot 2 \Pi(0.7)}\right)$ & $\left(0.6 \mathrm{e}^{i \cdot 2 \Pi(0.3)}, 0.5 \mathrm{e}^{i \cdot 2 \Pi(0.6)}\right)$ & $\left(0.6 \mathrm{e}^{i \cdot 2 \Pi(0.7)}, 0.6 \mathrm{e}^{i \cdot 2 \Pi(0.3)}\right)$ & $\left(0.7 \mathrm{e}^{i \cdot 2 \Pi(0.6)}, 0.5 \mathrm{e}^{i \cdot 2 \Pi(0.2)}\right)$ & $\left(1 \cdot e^{i \cdot 2 \Pi}, 0\right)$ \\
$x_{6}$ & $\left(0.4 \mathrm{e}^{i \cdot 2 \Pi(0.6)}, 0.7 \mathrm{e}^{i \cdot 2 \Pi(0.6)}\right)$ & $\left(0.2 \mathrm{e}^{i \cdot 2 \Pi(0.7)}, 0.8 \mathrm{e}^{i \cdot 2 \Pi(0.2)}\right)$ & $\left(0.8 \mathrm{e}^{i \cdot 2 \Pi(0.6)}, 0.3 \mathrm{e}^{i \cdot 2 \Pi(0.5)}\right)$ & $\left(0.6 \mathrm{e}^{i \cdot 2 \Pi(0.8)}, 0.5 \mathrm{e}^{i \cdot 2 \Pi(0.3)}\right)$ & $\left(1 \cdot \mathrm{e}^{i \cdot 2 \Pi}, 0\right)$ \\
$x_{7}$ & $\left(0.2 \mathrm{e}^{i \cdot 2 \Pi(0.2)}, 0.5 \mathrm{e}^{i \cdot 2 \Pi(0.8)}\right)$ & $\left(0.1 \mathrm{e}^{i \cdot 2 \Pi(0.6)}, 0.9 \mathrm{e}^{i \cdot 2 \Pi(0.5)}\right)$ & $\left(0.2 \mathrm{e}^{i \cdot 2 \Pi(0.5)}, 0.8 \mathrm{e}^{i \cdot 2 \Pi(0.5)}\right)$ & $\left(0.8 \mathrm{e}^{i \cdot 2 \Pi(0.7)}, 0.3 \mathrm{e}^{i \cdot 2 \Pi(0.4)}\right)$ & $\left(1 \cdot \mathrm{e}^{i \cdot 2 \Pi}, 0\right)$ \\
\hline
\end{tabular}

Table 3 Distance measures of known and unknown quantities using $W D_{\mathrm{CPyFS}}^{1}$

Table 4 Distance measures of known and unknown quantities using $W D_{\mathrm{CPyFS}}^{2}$

\begin{tabular}{llll}
\hline$W D_{\mathrm{CPyFS}}^{1}\left(A_{1}, A\right)$ & $W D_{\mathrm{CPyFS}}^{1}\left(A_{2}, A\right)$ & $W D_{\mathrm{CPyFS}}^{1}\left(A_{3}, A\right)$ & $W D_{\mathrm{CPyFS}}^{1}\left(A_{4}, A\right)$ \\
\hline 0.568 & 0.077 & 0.079 & 0.067 \\
\hline
\end{tabular}

\begin{tabular}{llll}
\hline$W D_{\mathrm{CPyFS}}^{2}\left(A_{1}, A\right)$ & $W D_{\mathrm{CPyFS}}^{2}\left(A_{2}, A\right)$ & $W D_{\mathrm{CPyFS}}^{2}\left(A_{3}, A\right)$ & $W D_{\mathrm{CPyFS}}^{2}\left(A_{4}, A\right)$ \\
\hline 0.270 & 0.282 & 0.275 & 0.288 \\
\hline
\end{tabular}

Table 5 Ranking of distance measures

\begin{tabular}{ll}
\hline & Ranking \\
\hline Using Eq. (3) & $A_{1}>A_{3}>A_{2}>A_{4}$ \\
Using Eq. (4) & $A_{4}>A_{2}>A_{3}>A_{1}$ \\
\hline
\end{tabular}

and $\left\{a_{2}=0.1, b_{2}=0.2, c_{2}=0.6, r_{2}=0.1\right\}$ are provided in Table 4.

3. This step involves the ranking of distance measures of obtained using Eqs. (3) and (4). The ranking of known building materials based on distance measures is provided in Table 5. The unknown building material belongs to the class with which its distance measure is the least.

The analysis of information obtained in Table 5 shows that using the weighted distance measure defined in Eq. (3), the class of unknown building material is $A_{4}$, i.e., the unknown building material belongs to the class of polyvinyl chloride flooring. This is because the unknown building material has least distance measure with polyvinyl chloride flooring. On the other hand, if we use the distance measure defined in Eq. (4), the unknown building material seems to belong to class $A_{1}$, i.e., sealant due to its least distance measure value with $A_{1}$. The results obtained using Eq. (4) are considered as more accurate due to the fact that the distance measure defined in Eq. (4) takes into account the hesitancy degree of the information along with membership and nonmembership values.

\section{Comparative study}

In this section, we established the comparison of the proposed distance measures of CPFSs with PFS, CIFS, IFS, CFS, and FS. With the help of some restrictions on the proposed DMs, it is proposed that these DMs reduce the environments of PFS, CIFS, IFS, CFS, and FS. The comparison is demonstrated in Remarks $1-5$. We have also considered the numerical data in other fuzzy environments and show applicability of the proposed work distance measures in those situations.

Remark 1 The DMs proposed in Eqs. (3) and (4) for CPFSs reduce to the environment of PFSs if we considered the imaginary part as zero as defined in Eqs. (5) and (6):

$$
\begin{aligned}
W & D_{\mathrm{PyFS}}^{1}(A, B) \\
= & \frac{1}{2 \sum_{i=1}^{n} \omega_{i}}\left[\sum _ { i = 1 } ^ { n } \omega _ { i } \left[\left(a_{1} \cdot\left|T_{A}^{2}\left(x_{i}\right)-T_{B}^{2}\left(x_{i}\right)\right|\right.\right.\right. \\
& +b_{1} \cdot\left|F_{A}^{2}\left(x_{i}\right)-F_{B}^{2}\left(x_{i}\right)\right|+c_{1} \cdot \max \left(\left|T_{A} s s^{2}\left(x_{i}\right)-T_{B}^{2}\left(x_{i}\right)\right|,\right. \\
& \left.\left.\left.\left.\left|F_{A}^{2}\left(x_{i}\right)-F_{B}^{2}\left(x_{i}\right)\right|\right)\right)\right]\right]
\end{aligned}
$$

$$
\begin{aligned}
& W D_{\mathrm{PyFS}}^{2}(A, B) \\
& =\frac{1}{2 \sum_{i=1}^{n} \omega_{i}}\left[\sum _ { i = 1 } ^ { n } \omega _ { i } \left[\left(a_{1} \cdot\left|T_{A}^{2}\left(x_{i}\right)-T_{B}^{2}\left(x_{i}\right)\right|\right.\right.\right. \\
& \quad+b_{1} \cdot\left|F_{A}^{2}\left(x_{i}\right)-F_{B}^{2}\left(x_{i}\right)\right|+r_{1} \cdot\left|R_{A}^{2}\left(x_{i}\right)-R_{B}^{2}\left(x_{i}\right)\right|
\end{aligned}
$$




$$
\begin{aligned}
& +c_{1} \cdot \max \left(\left|T_{A}^{2}\left(x_{i}\right)-T_{B}^{2}\left(x_{i}\right)\right|,\left|F_{A}^{2}\left(x_{i}\right)-F_{B}^{2}\left(x_{i}\right)\right|,\right. \\
& \left.\left.\left.\left.\left|R_{A}^{2}\left(x_{i}\right)-R_{B}^{2}\left(x_{i}\right)\right|\right)\right)\right]\right] .
\end{aligned}
$$

Remark 2 The DMs proposed in Eqs. (3) and (4) for CPFSs reduce to the environment of CIFSs if the constraint $0 \leq$ $\left|z_{1}\right|^{2}+\left|z_{2}\right|^{2} \leq 1$ is replaced by $0 \leq\left|z_{1}\right|+\left|z_{2}\right| \leq 1$, i.e., 2 is replaced by 1, as defined in Eqs. (7) and (8):

$$
\begin{aligned}
& W D_{\mathrm{CIFS}}^{1}(A, B)=\frac{1}{2 \sum_{i=1}^{n} \omega_{i}} \\
& \qquad \sum_{i=1}^{n} \omega_{i}\left[\left(a_{1} \cdot\left|T_{A}\left(x_{i}\right)-T_{B}\left(x_{i}\right)\right|+b_{1} \cdot\left|F_{A}\left(x_{i}\right)-F_{B}\left(x_{i}\right)\right|\right.\right. \\
& \left.\quad+c_{1} \cdot \max \left(\left|T_{A}\left(x_{i}\right)-T_{B}\left(x_{i}\right)\right|,\left|F_{A}\left(x_{i}\right)-F_{B}\left(x_{i}\right)\right|\right)\right) \\
& \quad+\frac{1}{2 \Pi}\left(a_{2} \cdot\left|2 \Pi W_{T_{A}}\left(x_{i}\right)-2 \Pi W_{T_{B}}\left(x_{i}\right)\right|\right. \\
& \quad+b_{2} \cdot\left|2 \Pi W_{F_{A}}\left(x_{i}\right)-2 \Pi W_{F_{B}}\left(x_{i}\right)\right| \\
& \quad+c_{2} \cdot \max \left(\left|2 \Pi W_{T_{A}}\left(x_{i}\right)-2 \Pi W_{T_{B}}\left(x_{i}\right)\right|\right. \\
& \left.\left.\left.\left.\quad\left|2 \Pi W_{F_{A}}\left(x_{i}\right)-2 \Pi W_{F_{B}}\left(x_{i}\right)\right|\right)\right)\right]\right]
\end{aligned}
$$$$
W D_{\mathrm{CIFS}}^{2}(A, B)
$$$$
=\frac{1}{2 \sum_{i=1}^{n} \omega_{i}}\left[\sum _ { i = 1 } ^ { n } \omega _ { i } \left[\left(a_{1} \cdot\left|T_{A}\left(x_{i}\right)-T_{B}\left(x_{i}\right)\right|\right.\right.\right.
$$$$
+b_{1} \cdot\left|F_{A}\left(x_{i}\right)-F_{B}\left(x_{i}\right)\right|+r_{1} \cdot\left|R_{A}\left(x_{i}\right)-R_{B}\left(x_{i}\right)\right|
$$$$
+c_{1} \cdot \max \left(\left|T_{A}\left(x_{i}\right)-T_{B}\left(x_{i}\right)\right|\right. \text {, }
$$$$
\left.\left.\left|F_{A}\left(x_{i}\right)-F_{B}\left(x_{i}\right)\right|,\left|R_{A}\left(x_{i}\right)-R_{B}\left(x_{i}\right)\right|\right)\right)
$$$$
+\frac{1}{2 \Pi}\left(a_{2} \cdot\left|2 \Pi W_{T_{A}}\left(x_{i}\right)-2 \Pi W_{T_{B}}\left(x_{i}\right)\right|\right.
$$$$
+b_{2} \cdot\left|2 \Pi W_{F_{A}}\left(x_{i}\right)-2 \Pi W_{F_{B}}\left(x_{i}\right)\right|
$$$$
+r_{2} \cdot\left|2 \Pi W_{R_{A}}\left(x_{i}\right)-2 \Pi W_{R_{B}}\left(x_{i}\right)\right|
$$$$
+c_{2} \cdot \max \left(\left|2 \Pi W_{T_{A}}\left(x_{i}\right)-2 \Pi W_{T_{B}}\left(x_{i}\right)\right|\right. \text {, }
$$$$
\left|2 \Pi W_{F_{A}}\left(x_{i}\right)-2 \Pi W_{F_{B}}\left(x_{i}\right)\right| \text {, }
$$$$
\left.\left.\left.\left.\left|2 \Pi W_{R_{A}}\left(x_{i}\right)-2 \Pi W_{R_{B}}\left(x_{i}\right)\right|\right)\right)\right]\right] \text {. }
$$

Remark 3 The DMs proposed in Eqs. (3) and (4) for CPFSs reduce to the environment of IFSs as defined in Eqs. (9) and (10).

$$
\begin{aligned}
& W D_{\mathrm{CPyFS}}^{1}(A, B) \\
& =\frac{1}{2 \sum_{i=1}^{n} \omega_{i}}\left[\sum _ { i = 1 } ^ { n } \omega _ { i } \left[\left(a_{1} \cdot\left|T_{A}\left(x_{i}\right)-T_{B}\left(x_{i}\right)\right|\right.\right.\right. \\
& \quad+b_{1} \cdot\left|F_{A}\left(x_{i}\right)-F_{B}\left(x_{i}\right)\right| \\
& \left.\left.\left.\quad+c_{1} \cdot \max \left(\left|T_{A}\left(x_{i}\right)-T_{B}\left(x_{i}\right)\right|,\left|F_{A}\left(x_{i}\right)-F_{B}\left(x_{i}\right)\right|\right)\right)\right]\right]
\end{aligned}
$$

$$
\begin{aligned}
& W D_{\mathrm{CPyFS}}^{2}(A, B) \\
& =\frac{1}{2 \sum_{i=1}^{n} \omega_{i}}\left[\sum _ { i = 1 } ^ { n } \omega _ { i } \left[\left(a_{1} \cdot\left|T_{A}\left(x_{i}\right)-T_{B}\left(x_{i}\right)\right|\right.\right.\right. \\
& \quad+b_{1} \cdot\left|F_{A}\left(x_{i}\right)-F_{B}\left(x_{i}\right)\right|+r_{1} \cdot\left|R_{A}\left(x_{i}\right)-R_{B}\left(x_{i}\right)\right| \\
& \quad+c_{1} \cdot \max \left(\left|T_{A}\left(x_{i}\right)-T_{B}\left(x_{i}\right)\right|,\left|F_{A}\left(x_{i}\right)-F_{B}\left(x_{i}\right)\right|,\right. \\
& \\
& \left.\left.\left.\left.\quad\left|R_{A}\left(x_{i}\right)-R_{B}\left(x_{i}\right)\right|\right)\right)\right]\right]
\end{aligned}
$$

Remark 4 The DMs proposed in Eqs. (3) and (4) for CPFSs reduce to the environment of CFSs as defined in Eqs. (11) and (12) [6]:

$$
\begin{aligned}
& W D_{\mathrm{CFS}}^{1}(A, B) \\
& =\frac{1}{2 \sum_{i=1}^{n} \omega_{i}}\left[\sum _ { i = 1 } ^ { n } \omega _ { i } \left[\left(a_{1} \cdot\left|T_{A}\left(x_{i}\right)-T_{B}\left(x_{i}\right)\right|\right.\right.\right. \\
& \left.\quad+c_{1} \cdot \max \left(\left|T_{A}\left(x_{i}\right)-T_{B}\left(x_{i}\right)\right|\right)\right) \\
& \quad+\frac{1}{2 \Pi}\left(a_{2} \cdot\left|2 \Pi W_{T_{A}}\left(x_{i}\right)-2 \Pi W_{T_{B}}\left(x_{i}\right)\right|\right. \\
& \left.\left.\quad+c_{2} \cdot \max \left(\left|2 \Pi W_{T_{A}}\left(x_{i}\right)-2 \Pi W_{T_{B}}\left(x_{i}\right)\right|\right)\right)\right]
\end{aligned}
$$

$W D_{\mathrm{CFS}}^{2}(A, B)$

$$
\begin{aligned}
= & \frac{1}{2 \sum_{i=1}^{n} \omega_{i}}\left[\sum _ { i = 1 } ^ { n } \omega _ { i } \left[\left(a_{1} \cdot\left|T_{A}\left(x_{i}\right)-T_{B}\left(x_{i}\right)\right|\right.\right.\right. \\
& +r_{1} \cdot\left|R_{A}\left(x_{i}\right)-R_{B}\left(x_{i}\right)\right| \\
& \left.+c_{1} \cdot \max \left(\left|T_{A}\left(x_{i}\right)-T_{B}\left(x_{i}\right)\right|,\left|R_{A}\left(x_{i}\right)-R_{B}\left(x_{i}\right)\right|\right)\right) \\
& +\frac{1}{2 \Pi}\left(a_{2} \cdot\left|2 \Pi W_{T_{A}}\left(x_{i}\right)-2 \Pi W_{T_{B}}\left(x_{i}\right)\right|\right. \\
& +r_{2} \cdot\left|2 \Pi W_{R_{A}}\left(x_{i}\right)-2 \Pi W_{R_{B}}\left(x_{i}\right)\right| \\
& +c_{2} \cdot \max \left(\left|2 \Pi W_{T_{A}}\left(x_{i}\right)-2 \Pi W_{T_{B}}\left(x_{i}\right)\right|,\right. \\
& \left.\left.\left.\left.\left|2 \Pi W_{R_{A}}\left(x_{i}\right)-2 \Pi W_{R_{B}}\left(x_{i}\right)\right|\right)\right)\right]\right] .
\end{aligned}
$$

Remark 5 The DMs proposed in Eqs. (3) and (4) for CPFSs reduce to the environment of IFSs as defined in Eqs. (13) and (14) [29]:

$$
\begin{aligned}
W D_{\mathrm{CFS}}^{1}(A, B)= & \frac{1}{2 \sum_{i=1}^{n} \omega_{i}}\left[\sum _ { i = 1 } ^ { n } \omega _ { i } \left[\left(a_{1} \cdot\left|T_{A}\left(x_{i}\right)-T_{B}\left(x_{i}\right)\right|\right.\right.\right. \\
& \left.\left.\left.+c_{1} \cdot \max \left(\left|T_{A}\left(x_{i}\right)-T_{B}\left(x_{i}\right)\right|\right)\right)\right]\right]
\end{aligned}
$$

$W D_{\mathrm{CFS}}^{2}(A, B)$

$$
=\frac{1}{2 \sum_{i=1}^{n} \omega_{i}}\left[\sum _ { i = 1 } ^ { n } \omega _ { i } \left[\left(a_{1} \cdot\left|T_{A}\left(x_{i}\right)-T_{B}\left(x_{i}\right)\right|\right.\right.\right.
$$


Table 6 Complex intuitionistic fuzzy information about known and unknown materials

\begin{tabular}{|c|c|c|c|c|c|}
\hline & $A_{1}$ & $A_{2}$ & $A_{3}$ & $A_{4}$ & $A$ \\
\hline$x_{1}$ & $\left(0.7 \mathrm{e}^{i \cdot 2 \Pi(0.1)}, 0.3 \mathrm{e}^{i \cdot 2 \Pi(0.4)}\right)$ & $\left(0.6 e^{i .2 \Pi(0.2)}, 0.4 e^{i .2 \Pi(0.5)}\right)$ & $\left(0.5 \mathrm{e}^{i \cdot 2 \Pi(0.2)}, 0.4 \mathrm{e}^{i \cdot 2 \Pi(0.4)}\right)$ & $\left(0.7 \mathrm{e}^{i \cdot 2 \Pi(0.6)}, 0.3 \mathrm{e}^{i \cdot 2 \Pi(0.3)}\right)$ & $\left(1 \cdot \mathrm{e}^{i \cdot 2 \Pi}, 0\right)$ \\
\hline$x_{2}$ & $\left(0.3 \mathrm{e}^{i \cdot 2 \Pi(0.4)}, 0.5 \mathrm{e}^{i \cdot 2 \Pi(0.6)}\right)$ & $\left(0.4 \mathrm{e}^{i \cdot 2 \Pi(0.3)}, 0.6 \mathrm{e}^{i \cdot 2 \Pi(0.4)}\right)$ & $\left(0.6 \mathrm{e}^{i \cdot 2 \Pi(0.5)}, 0.3 \mathrm{e}^{i \cdot 2 \Pi(0.5)}\right)$ & $\left(0.6 \mathrm{e}^{i \cdot 2 \Pi(0.4)}, 0.4 \mathrm{e}^{i \cdot 2 \Pi(0.2)}\right)$ & $\left(1 \cdot \mathrm{e}^{i \cdot 2 \Pi}, 0\right)$ \\
\hline$x_{3}$ & $\left(0.8 \mathrm{e}^{i \cdot 2 \Pi(0.3)}, 0.2 \mathrm{e}^{i \cdot 2 \Pi(0.3)}\right)$ & $\left(0.7 \mathrm{e}^{i \cdot 2 \Pi(0.5)}, 0.3 \mathrm{e}^{i \cdot 2 \Pi(0.3)}\right)$ & $\left(0.3 \mathrm{e}^{i \cdot 2 \Pi(0.1)}, 0.3 \mathrm{e}^{i \cdot 2 \Pi(0.7)}\right)$ & $\left(0.5 \mathrm{e}^{i .2 \Pi(0.3)}, 0.5 \mathrm{e}^{i \cdot 2 \Pi(0.6)}\right)$ & $\left(1 \cdot \mathrm{e}^{i \cdot 2 \Pi}, 0\right)$ \\
\hline$x_{4}$ & $\left(0.5 \mathrm{e}^{i \cdot 2 \Pi(0.4)}, 0.5 \mathrm{e}^{i \cdot 2 \Pi(0.3)}\right)$ & $\left(0.6 \mathrm{e}^{i \cdot 2 \Pi(0.7)}, 0.3 \mathrm{e}^{i \cdot 2 \Pi(0.2)}\right)$ & $\left(0.5 \mathrm{e}^{i \cdot 2 \Pi(0.2)}, 0.5 \mathrm{e}^{i \cdot 2 \Pi(0.3)}\right)$ & $\left(0.4 \mathrm{e}^{i \cdot 2 \Pi(0.2)}, 0.5 \mathrm{e}^{i \cdot 2 \Pi(0.2)}\right)$ & $\left(1 \cdot \mathrm{e}^{i \cdot 2 \Pi}, 0\right)$ \\
\hline$x_{5}$ & $\left(0.5 \mathrm{e}^{i \cdot 2 \Pi(0.4)}, 0.4 \mathrm{e}^{i \cdot 2 \Pi(0.4)}\right)$ & $\left(0.6 \mathrm{e}^{i \cdot 2 \Pi(0.1)}, 0.4 \mathrm{e}^{i \cdot 2 \Pi(0.2)}\right)$ & $\left(0.6 \mathrm{e}^{i \cdot 2 \Pi(0.7)}, 0.3 \mathrm{e}^{i \cdot 2 \Pi(0.1)}\right)$ & $\left(0.4 \mathrm{e}^{i \cdot 2 \Pi(0.4)}, 0.5 \mathrm{e}^{i \cdot 2 \Pi(0.1)}\right)$ & $\left(1 \cdot \mathrm{e}^{i \cdot 2 \Pi}, 0\right)$ \\
\hline$x_{6}$ & $\left(0.4 \mathrm{e}^{i \cdot 2 \Pi(0.8)}, 0.5 \mathrm{e}^{i \cdot 2 \Pi(0.1)}\right)$ & $\left(0.2 \mathrm{e}^{i \cdot 2 \Pi(0.2)}, 0.8 \mathrm{e}^{i \cdot 2 \Pi(0.4)}\right)$ & $\left(0.7 \mathrm{e}^{i \cdot 2 \Pi(0.4)}, 0.3 \mathrm{e}^{i \cdot 2 \Pi(0.3)}\right)$ & $\left(0.4 \mathrm{e}^{i \cdot 2 \Pi(0.4)}, 0.5 \mathrm{e}^{i \cdot 2 \Pi(0.2)}\right)$ & $\left(1 \cdot \mathrm{e}^{i \cdot 2 \Pi}, 0\right)$ \\
\hline$x_{7}$ & $\left(0.2 \mathrm{e}^{i \cdot 2 \Pi(0.6)}, 0.5 \mathrm{e}^{i \cdot 2 \Pi(0.3)}\right)$ & $\left(0.1 \mathrm{e}^{i \cdot 2 \Pi(0.4)}, 0.9 \mathrm{e}^{i \cdot 2 \Pi(0.6)}\right)$ & $\left(0.2 \mathrm{e}^{i \cdot 2 \Pi(0.4)}, 0.8 \mathrm{e}^{i \cdot 2 \Pi(0.4)}\right)$ & $\left(0.7 \mathrm{e}^{i \cdot 2 \Pi(0.4)}, 0.3 \mathrm{e}^{i \cdot 2 \Pi(0.5)}\right)$ & $\left(1 \cdot \mathrm{e}^{i \cdot 2 \Pi}, 0\right)$ \\
\hline \multirow{2}{*}{\multicolumn{2}{|c|}{$\begin{array}{l}\text { Table } 7 \text { Distance measures of } \\
\text { known and unknown quantities }\end{array}$}} & $W D_{\mathrm{CPyFS}}^{1}\left(A_{1}, A\right)$ & $W D_{\mathrm{CPyFS}}^{1}\left(A_{2}, A\right)$ & $D_{\mathrm{CPyFS}}^{1}\left(A_{3}, A\right)$ & $W D_{\mathrm{CPyFS}}^{1}\left(A_{4}, A\right)$ \\
\hline & & 0.564 & 0.0936 & 0.08669 & 0.08985 \\
\hline \multirow{2}{*}{\multicolumn{2}{|c|}{$\begin{array}{l}\text { Table } 8 \text { Distance measures of } \\
\text { known and unknown quantities }\end{array}$}} & $W D_{\mathrm{CPyFS}}^{2}\left(A_{1}, A\right)$ & $W D_{\mathrm{CPyFS}}^{2}\left(A_{2}, A\right)$ & $W D_{\mathrm{CPyFS}}^{2}\left(A_{3}, A\right)$ & $W D_{\mathrm{CPyFS}}^{2}\left(A_{4}, A\right)$ \\
\hline & & 0.02835 & 0.314 & 0.294 & 0.307 \\
\hline
\end{tabular}

Table 9 Ranking of distance measures

\begin{tabular}{ll}
\hline & Ranking \\
\hline Using Eq. (3) & $A_{3}>A_{4}>A_{2}>A_{1}$ \\
Using Eq. (4) & $A_{1}>A_{3}>A_{4}>A_{2}$ \\
\hline
\end{tabular}

$$
\begin{aligned}
& +r_{1} \cdot\left|R_{A}\left(x_{i}\right)-R_{B}\left(x_{i}\right)\right| \\
& \left.\left.\left.+c_{1} \cdot \max \left(\left|T_{A}\left(x_{i}\right)-T_{B}\left(x_{i}\right)\right|,\left|R_{A}\left(x_{i}\right)-R_{B}\left(x_{i}\right)\right|\right)\right)\right]\right] .
\end{aligned}
$$

All these results lead us to the point that the proposed distance measure can be applied to existing problems. Now, we consider the building material problem in the environment of CIFSs. The information of DMs is given in Table 6.

The distance measure proposed in Eq. (3) with $\left\{a_{1}=0.3\right.$, $\left.b_{1}=0.5, c_{1}=0.2\right\}$ and $\left\{a_{2}=0.1, b_{2}=0.2, c_{2}=0.7\right\}$ is applied to the data presented in Table 6 and the results are given in Table 7.

The distance measure proposed in Eq. (4) with $\left\{a_{1}=0.3\right.$, $\left.b_{1}=0.4, c_{1}=0.2, r_{1}=0.1\right\}$ and $\left\{a_{2}=0.1, b_{2}=0.2\right.$, $\left.c_{2}=0.6, r_{2}=0.1\right\}$ is applied to the data presented in Table 6 and the results are given in Table 8.

The ranking of distance measures obtained in Tables 7 and 8 is provided in Table 9.

Hence, the proposed distance measures are successfully applied to problem in the environment of CIFS. Similarly, these distance measures can also be applied to other fuzzy frameworks which are illustrated in Sect. 7. On the other
Table 10 Pythagorean fuzzy information about known and unknown materials

\begin{tabular}{llllll}
\hline & $A_{1}$ & $A_{2}$ & $A_{3}$ & $A_{4}$ & $A$ \\
\hline$x_{1}$ & $(0.8,0.3)$ & $(0.7,0.4)$ & $(0.5,0.7)$ & $(0.7,0.3)$ & $(1,0)$ \\
$x_{2}$ & $(0.7,0.5)$ & $(0.6,0.6)$ & $(0.6,0.5)$ & $(0.8,0.4)$ & $(1,0)$ \\
$x_{3}$ & $(0.9,0.2)$ & $(0.8,0.3)$ & $(0.3,0.3)$ & $(0.7,0.5)$ & $(1,0)$ \\
$x_{4}$ & $(0.6,0.5)$ & $(0.8,0.3)$ & $(0.5,0.5)$ & $(0.4,0.7)$ & $(1,0)$ \\
$x_{5}$ & $(0.5,0.6)$ & $(0.6,0.5)$ & $(0.6,0.6)$ & $(0.7,0.5)$ & $(1,0)$ \\
$x_{6}$ & $(0.4,0.7)$ & $(0.2,0.8)$ & $(0.8,0.3)$ & $(0.6,0.5)$ & $(1,0)$ \\
$x_{7}$ & $(0.2,0.5)$ & $(0.1,0.9)$ & $(0.2,0.8)$ & $(0.8,0.3)$ & $(1,0)$ \\
\hline
\end{tabular}

hand, none of the existing tools can be applied to problems lying in the environment of CPFSs.

\section{Advantages}

In this section, we demonstrate the advantages of working in the area of CPFS and DMs of CPFSs. Our claim is that the proposed distance measures can solve the problem lies in the region of PFSs, CIFSs, IFSs, CFSs, and FS. On the other hand, the DMs of PFSs, CIFSs, IFSs, CFSs, and FS could not handle the information provided in the form of CPFSs. We prove our claim with the help of some examples.

Suppose we have information about building materials in the form of PFSs, as shown in Table 10. Then, such problem can be solved using the restricted version of DMs proposed in Remark 1.

Suppose we have information about building materials in the form of IFSs, as shown in Table 11. Then, such problem 
Table 11 Intuitionistic fuzzy information about unknown and known materials

\begin{tabular}{llllll}
\hline & $A_{1}$ & $A_{2}$ & $A_{3}$ & $A_{4}$ & $A$ \\
\hline$x_{1}$ & $(0.7,0.3)$ & $(0.6,0.4)$ & $(0.5,0.4)$ & $(0.7,0.3)$ & $(1,0)$ \\
$x_{2}$ & $(0.3,0.5)$ & $(0.4,0.6)$ & $(0.6,0.3)$ & $(0.6,0.4)$ & $(1,0)$ \\
$x_{3}$ & $(0.8,0.2)$ & $(0.7,0.3)$ & $(0.3,0.3)$ & $(0.5,0.5)$ & $(1,0)$ \\
$x_{4}$ & $(0.5,0.5)$ & $(0.6,0.3)$ & $(0.5,0.5)$ & $(0.4,0.5)$ & $(1,0)$ \\
$x_{5}$ & $(0.5,0.4)$ & $(0.6,0.4)$ & $(0.6,0.3)$ & $(0.4,0.5)$ & $(1,0)$ \\
$x_{6}$ & $(0.4,0.5)$ & $(0.2,0.8)$ & $(0.7,0.3)$ & $(0.4,0.5)$ & $(1,0)$ \\
$x_{7}$ & $(0.2,0.5)$ & $(0.1,0.9)$ & $(0.2,0.8)$ & $(0.7,0.3)$ & $(1,0)$ \\
\hline
\end{tabular}

Table 12 Complex fuzzy information about unknown and known materials

\begin{tabular}{llllll}
\hline & $A_{1}$ & $A_{2}$ & $A_{3}$ & $A_{4}$ & $A$ \\
\hline$x_{1}$ & $0.7 \mathrm{e}^{i \cdot 2 \Pi(0.4)}$ & $0.6 \mathrm{e}^{i \cdot 2 \Pi(0.6)}$ & $0.5 \mathrm{e}^{i \cdot 2 \Pi(0.2)}$ & $0.7 \mathrm{e}^{i \cdot 2 \Pi(0.2)}$ & $1 \cdot \mathrm{e}^{i \cdot 2 \Pi}$ \\
$x_{2}$ & $0.3 \mathrm{e}^{i \cdot 2 \Pi(0.5)}$ & $0.4 \mathrm{e}^{i \cdot 2 \Pi(0.4)}$ & $0.6 \mathrm{e}^{i \cdot 2 \Pi(0.3)}$ & $0.6 \mathrm{e}^{i \cdot 2 \Pi(0.4)}$ & $1 \cdot \mathrm{e}^{i \cdot 2 \Pi}$ \\
$x_{3}$ & $0.8 \mathrm{e}^{i \cdot 2 \Pi(0.7)}$ & $0.7 \mathrm{e}^{i \cdot 2 \Pi(0.1)}$ & $0.3 \mathrm{e}^{i \cdot 2 \Pi(0.5)}$ & $0.5 \mathrm{e}^{i \cdot 2 \Pi(0.6)}$ & $1 \cdot \mathrm{e}^{i \cdot 2 \Pi}$ \\
$x_{4}$ & $0.5 e^{i .2 \Pi(0.9)}$ & $0.6 e^{i .2 \Pi(0.3)}$ & $0.5 e^{i .2 \Pi(0.7)}$ & $0.4 e^{i .2 \Pi(0.8)}$ & $1 . e^{i .2 \Pi}$ \\
$x_{5}$ & $0.5 \mathrm{e}^{i \cdot 2 \Pi(0.6)}$ & $0.6 \mathrm{e}^{i \cdot 2 \Pi(0.9)}$ & $0.6 \mathrm{e}^{i \cdot 2 \Pi(0.9)}$ & $0.4 \mathrm{e}^{i \cdot 2 \Pi(0.1)}$ & $1 \cdot \mathrm{e}^{i \cdot 2 \Pi}$ \\
$x_{6}$ & $0.4 \mathrm{e}^{i \cdot 2 \Pi(0.6)}$ & $0.2 \mathrm{e}^{i \cdot 2 \Pi(0.1)}$ & $0.7 \mathrm{e}^{i \cdot 2 \Pi(0.3)}$ & $0.4 \mathrm{e}^{i \cdot 2 \Pi(0.2)}$ & $1 \cdot \mathrm{e}^{i \cdot 2 \Pi}$ \\
$x_{7}$ & $0.2 \mathrm{e}^{i \cdot 2 \Pi(0.7)}$ & $0.1 \mathrm{e}^{i \cdot 2 \Pi(0.6)}$ & $0.2 \mathrm{e}^{i \cdot 2 \Pi(0.4)}$ & $0.7 \mathrm{e}^{i \cdot 2 \Pi(0.7)}$ & $1 \cdot \mathrm{e}^{i \cdot 2 \Pi}$ \\
\hline
\end{tabular}

can be solved using the restricted version of DMs proposed in Remark 3.

Suppose we have information about building materials in the form of CFSs as in Table 12. Then, such problem can be solved using the restricted version of DMs proposed in Remark 4.

On the other hand, if we consider the information provided in Table 2. Neither the DMs of PFS, nor of IFS, CIFS, and CFS could handle such type of data because of the limitation in their nature. All this discussion shows the superiority of our proposed work and the limitations of existing structures.

\section{Conclusion}

In this paper, a novel concept of CPFS is introduced due the limitations exist in the framework of CFS and CIFS. CFS and CIFS are critically examined and their limitations are pointed out numerically. The main contributions are:

1. A new definition for CIFS is proposed as well involving the degree of hesitancy.

2. The concept of CPFS is introduced and its novelty is discussed.

3. A geometrical comparison of CPFS is established with CFS and CIFS showing the superiority of CPFS.
4. Some distance measures for CPFSs are proposed and are applied to a building material recognition problem.

5. Comparative study of CPFS with CFS and CIFS is established and advantages of CPFS are studied.

In the near future, we aim to develop some aggregation operators for CPFS including weighted averaging and weighted geometric aggregation operators which can be utilized in MADM problems. The concept of power aggregation operators can also be established for CPFSs and utilized in MADM. A study of similarity and entropy measures is also suggested for future work.

\section{Compliance with ethical standards}

Conflict of interest The authors declared that they have no conflicts of interest regarding the publication of this manuscript.

Open Access This article is distributed under the terms of the Creative Commons Attribution 4.0 International License (http://creativecomm ons.org/licenses/by/4.0/), which permits unrestricted use, distribution, and reproduction in any medium, provided you give appropriate credit to the original author(s) and the source, provide a link to the Creative Commons license, and indicate if changes were made.

\section{References}

1. Molodtsov D (1999) Soft set theory-first results. Comput Math Appl 37(4-5):19-31

2. Pawlak Z (1982) Rough sets. Int J Comput Inform Sci 11(5):341-356

3. Zadeh LA (1965) Fuzzy sets. Inf Control 8(3):338-353

4. Atanassov KT (1986) Intuitionistic fuzzy sets. Fuzzy Sets Syst 20(1):87-96

5. Yager RR (2013) Pythagorean fuzzy subsets. In: IFSA world congress and NAFIPS annual meeting (IFSA/NAFIPS), 2013 joint, 2013, IEEE. https://doi.org/10.1109/ifsa-nafips.2013.6608375

6. Ramot D, Milo R, Friedman M, Kandel A (2002) Complex fuzzy sets. IEEE Trans Fuzzy Syst 10(2):171-186

7. Alkouri AS, Salleh AR (2012) Complex intuitionistic fuzzy sets. AIP Conf Proc 1482:464. https://doi.org/10.1063/1.4757515

8. Ma J, Zhang G, Lu J (2012) A method for multiple periodic factor prediction problems using complex fuzzy sets. IEEE Trans Fuzzy Syst 20(1):32-45

9. Dick S, Yager RR, Yazdanbakhsh O (2016) On Pythagorean and complex fuzzy set operations. IEEE Trans Fuzzy Syst 24(5):1009-1021

10. Liu L, Zhang X (2018) Comment on Pythagorean and complex fuzzy set operations. IEEE Trans Fuzzy Syst 26(6):3902-3904

11. Greenfield S, Chiclana F, Dick S (2016) Interval-valued complex fuzzy logic. In: Fuzzy systems (FUZZ-IEEE), 2016 IEEE international conference on, 2016, IEEE. https://doi.org/10.1109/fuzzieee.2016.7737939

12. Garg H, Rani D (2018) Some generalized complex intuitionistic fuzzy aggregation operators and their application to multicriteria decision-making process. Arab J Sci Eng. https://doi.org/10.1007/ s13369-018-3413-x 
13. Kumar T, Bajaj RK (2014) On complex intuitionistic fuzzy soft sets with distance measures and entropies. J Math. https://doi.org/ 10.1155/2014/972198 (Article ID 972198)

14. Rani D, Garg H (2018) Complex intuitionistic fuzzy power aggregation operators and their applications in multicriteria decisionmaking. Expert Syst. https://doi.org/10.1111/exsy.12325

15. Singh PK, Selvachandran G, Kumar CA (2019) Interval-valued complex fuzzy concept lattice and its granular decomposition. In: Recent developments in machine learning and data analytics, vol 714. Springer, Berlin, pp 275-283. https://doi.org/10.1007/978981-13-1280-9_26

16. Selvachandran G, Garg H, Quek SG (2018) Vague entropy measure for complex vague soft sets. Entropy 20(6):403. https://doi.org/10. 3390/e20060403

17. Hu B, Bi L, Dai S, Li S (2018) Distances of complex fuzzy sets and continuity of complex fuzzy operations. J Intell Fuzzy Syst 35(2):2247-2255

18. Selvachandran G, Garg H, Alaroud MH, Salleh AR (2018) Similarity measure of complex vague soft sets and its application to pattern recognition. Int J Fuzzy Syst 20(6):1901-1914

19. Quek SG, Selvachandran G (2018) The algebraic structures of complex intuitionistic fuzzy soft sets associated with groups and subgroups. Sci Iran. https://doi.org/10.24200/sci.2018.50050.1485

20. Feng L, Ma J, Wang Y, Yang J (2018) Comparison study on development path for small and medium-sized enterprises e-commerce using complex fuzzy sets. Int J Comput Intell Syst 11(1):716-724

21. Singh PK (2018) Complex fuzzy concept lattice. Neural Process Lett. https://doi.org/10.1007/s11063-018-9884-7

22. Selvachandran G, Singh PK (2018) Interval-valued complex fuzzy soft set and its application. Int J Uncertain Quant 8(2):101-117

23. Selvachandran G, Pal M, Alhawari TAA, Salleh AR (2018) Interval-valued complex fuzzy sets and its application to the malaysian economy. Int J Fuzzy Syst Appl (IJFSA) 7(1):22-31

24. Alsarahead MO, Ahmad AG (2018) Complex intuitionistic fuzzy ideals. In: AIP conference proceedings, 2018, AIP Publishing. https://doi.org/10.1063/1.5028033

25. Hu B, Bi L, Dai S, Li S (2018) The approximate parallelity of complex fuzzy sets. J Intell Fuzzy Syst 35(6):6343-6351

26. Ngan RT, Cuong BC, Ali M (2018) H-max distance measure of intuitionistic fuzzy sets in decision making. Appl Soft Comput 69:393-425. https://doi.org/10.1016/j.asoc.2018.04.036

27. Mishra AR, Singh RK, Motwani D (2018) Multi-criteria assessment of cellular mobile telephone service providers using intuitionistic fuzzy WASPAS method with similarity measures. Granul Comput. https://doi.org/10.1007/s41066-018-0114-5

28. Shen F, Ma X, Li Z, Xu Z, Cai D (2018) An extended intuitionistic fuzzy TOPSIS method based on a new distance measure with an application to credit risk evaluation. Inf Sci 428:105-119

29. Hwang CM, Yang MS, Hung WL (2018) New similarity measures of intuitionistic fuzzy sets based on the Jaccard index with its application to clustering. Int J Intell Syst 33(8):1672-1688

30. Luo M, Zhao R (2018) A distance measure between intuitionistic fuzzy sets and its application in medical diagnosis. Artif Intell Med 89:34-39. https://doi.org/10.1016/j.artmed.2018.05.002

31. Li D, Zeng W (2018) Distance measure of Pythagorean fuzzy sets. Int J Intell Syst 33(2):348-361

32. Wei G, Wei Y (2018) Similarity measures of Pythagorean fuzzy sets based on the cosine function and their applications. Int J Intell Syst 33(3):634-652

33. Zeng W, Li D, Yin Q (2018) Distance and similarity measures of Pythagorean fuzzy sets and their applications to multiple criteria group decision making. Int J Intell Syst 33(11):2236-2254
34. Biswas A, Sarkar B (2018) Pythagorean fuzzy multicriteria group decision making through similarity measure based on point operators. Int J Intell Syst 33(8):1731-1744

35. Garg H (2017) Distance and similarity measures for intuitionistic multiplicative preference relation and its applications. Int J Uncertain Quant 7(2):117-133

36. Garg H, Kumar K (2018) An advanced study on the similarity measures of intuitionistic fuzzy sets based on the set pair analysis theory and their application in decision making. Soft Comput 22:4959-4970

37. Garg H (2018) An improved cosine similarity measure for intuitionistic fuzzy sets and their applications to decision-making process. Hacet J Math Stat 47(6):1578-1594

38. Singh S, Garg H (2017) Distance measures between type-2 intuitionistic fuzzy sets and their application to multicriteria decisionmaking process. Appl Intell 46(4):788-799

39. Garg H, Kumar K (2018) Distance measures for connection number sets based on set pair analysis and its applications to decisionmaking process. Appl Intell 48(10):3346-3359

40. Ullah K, Mahmood T, Jan N (2018) Similarity measures for T-spherical fuzzy sets with applications in pattern recognition. Symmetry 10(6):193. https://doi.org/10.3390/sym10060193

41. Mahmood T, Ullah K, Khan Q (2018) Some aggregation operators for bipolar-valued hesitant fuzzy information. J Fundam Appl Sci 10(4S):240-245

42. Mahmood T, Ullah K, Ullah M, Jan N, Deli I (2017) Some aggregation operators for bipolar-valued hesitant fuzzy information based on einstein operational laws. J Eng Appl Sci 36(2):63-72

43. Ullah K, Mahmood T, Jan N, Broumi S, Khan Q (2018) On bipolarvalued hesitant fuzzy sets and their applications in multi-attribute decision making. Nucleus 55(2):85-93

44. Mahmood T, Ullah K, Khan Q, Jan N (2018) An approach toward decision-making and medical diagnosis problems using the concept of spherical fuzzy sets. Neural Comput Appl. https://doi.org/10. 1007/s00521-018-3521-2

45. Jan N, Zeedam L, Mahmood T, Ullah K, Ali Z (2018) Multiple attribute decision making method under linguistic cubic information. J Intell Fuzzy Syst. https://doi.org/10.3233/jifs-181253 (pre-press)

46. Peng X, Selvachandran G (2018) Pythagorean fuzzy set: state of the art and future directions. Artif Intell Rev. https://doi.org/10. 1007/s10462-017-9596-9

47. Peng X, Yuan H, Yang Y (2017) Pythagorean fuzzy information measures and their applications. Int J Intell Syst 32(10):991-1029

48. Peng X, Yang Y (2016) Pythagorean fuzzy Choquet integral based MABAC method for multiple attribute group decision making. Int J Intell Syst 31(10):989-1020

49. Peng X, Yang Y (2015) Some results for Pythagorean fuzzy sets. Int J Intell Syst 30(11):1133-1160

50. Garg H (2018) Hesitant Pythagorean fuzzy Maclaurin symmetric mean operators and its applications to multi-attribute decisionmaking process. Int J Intell Syst 34(4):601-626

51. Garg H (2019) New logarithmic operational laws and their aggregation operators for Pythagorean fuzzy set and their applications. Int J Intell Syst 34(1):82-106

52. Garg H, Arora R (2019) Generalized intuitionistic fuzzy soft power aggregation operator based on t-norm and their application in multicriteria decision-making. Int J Intell Syst 34(2):215-246

53. Garg H, Rani D (2019) A robust correlation coefficient measure of complex intuitionistic fuzzy sets and their applications in decisionmaking. Appl Intell 49(2):496-512

54. Garg H (2019) A hybrid GSA-GA algorithm for constrained optimization problems. Inf Sci 478:499-523. https://doi.org/10.1016/ j.ins.2018.11.041 
55. Garg H, Kumar K (2019) An advanced study on operations of connection number based on set pair analysis. Natl Acad Sci Lett. https://doi.org/10.1007/s40009-018-0748-5

56. Garg H (2019) Intuitionistic fuzzy hamacher aggregation operators with entropy weight and their applications to multi-criteria decision-making problems. Iran J Sci Technol Trans Electr Eng. https://doi.org/10.1007/s40998-018-0167-0
57. Garg H, Munir M, Ullah K, Mahmood T, Jan N (2018) Algorithm for T-spherical fuzzy multi-attribute decision making based on improved interactive aggregation operators. Symmetry 10(12):670. https://doi.org/10.3390/sym10120670

Publisher's Note Springer Nature remains neutral with regard to jurisdictional claims in published maps and institutional affiliations. 